\title{
Oriented attachment- and defect-dependent PbTe quantum dots growth: Shape transformations supported by Experimental insights and DFT calculations
}

\author{
Hugo Rojas-Chávez ${ }^{\text {a, }}$, Alan Miralrio ${ }^{\text {b }}$, Heriberto Cruz-Martínez ${ }^{\text {c }}$, Guillermo Carbajal- \\ Franco $^{\mathrm{d}}$, Manuel A. Valdés-Madrigal ${ }^{\mathrm{e}}$
}

a Tecnológico Nacional de México, Instituto Tecnológico de Tláhuac II, Departamento de Ingenierías, Camino Real 625, Col. Jardines del Llano, San Juan Ixtayopan. Alcaldía Tláhuac, CDMX 13508, Mexico.

b Tecnologico de Monterrey, Escuela de Ingeniería y Ciencias, Ave. Eugenio Garza Sada 2501, Monterrey 64849, N.L., Mexico.

${ }^{\mathrm{c}}$ Tecnológico Nacional de México, Instituto Tecnológico del Valle de Etla, Abasolo S/N, Barrio del Agua Buena, Santiago Suchilquitongo, Oaxaca 68230, Mexico.

d Tecnológico Nacional de México, Instituto Tecnológico de Toluca, Division of Graduate Studies and Research, Av. Tecnológico s.n. Metepec Estado de México 52149, Mexico.

e Tecnológico Nacional de México, Instituto Tecnológico Superior de Ciudad Hidalgo, Av. Ing. Carlos Rojas Gutiérrez 2120, Fracc. Valle de la herradura, Michoacán 61100, Mexico.

* Corresponding author: (H. Rojas-Chávez) rojas_hugo@ittlahuac2.edu.mx, huroch@gmail.com 


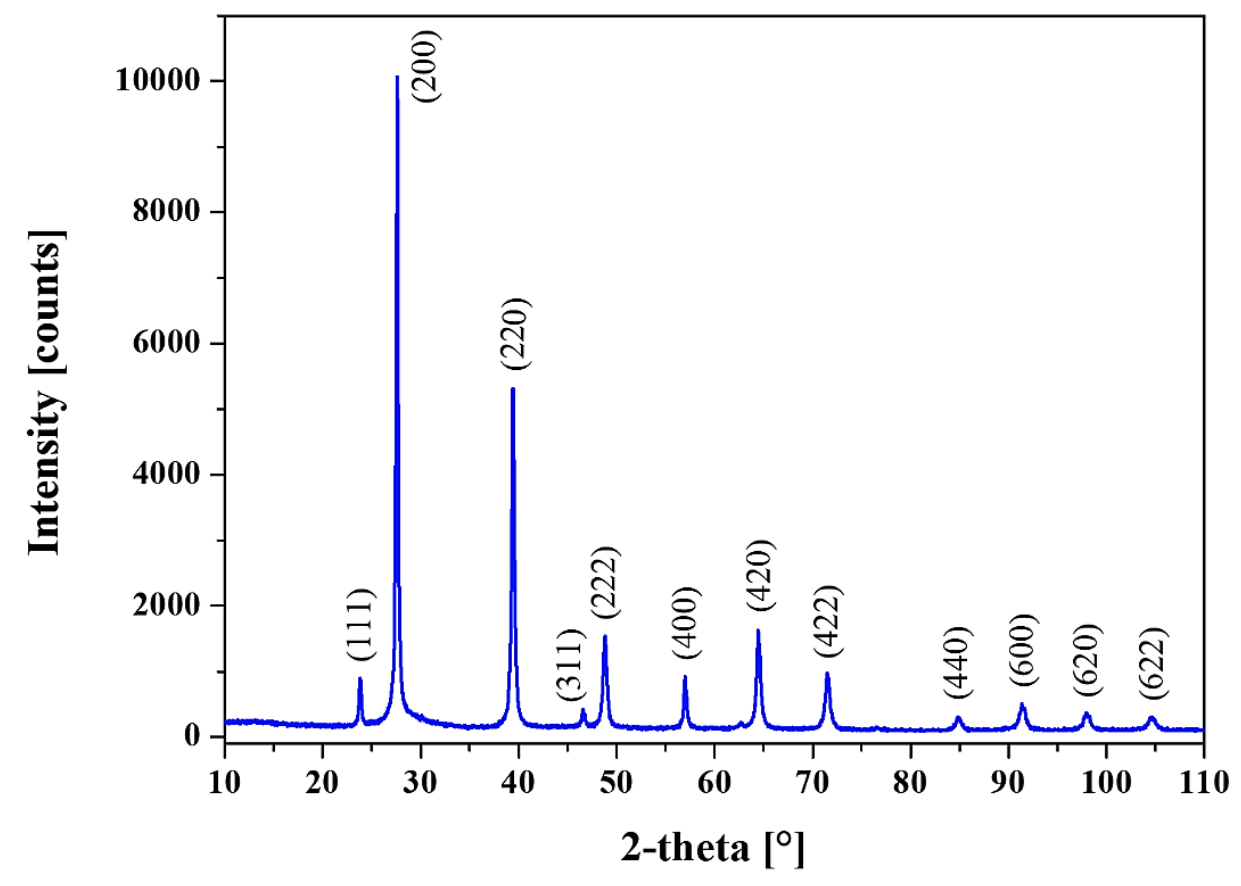

Figure S1. XRD pattern of PbTe QDs synthesized, for $5 \mathrm{~h}$ of milling, in the presence of formaldehyde. The Miller indices on the upper diffraction pattern correspond to the $\mathrm{PbTe}$ compound. 


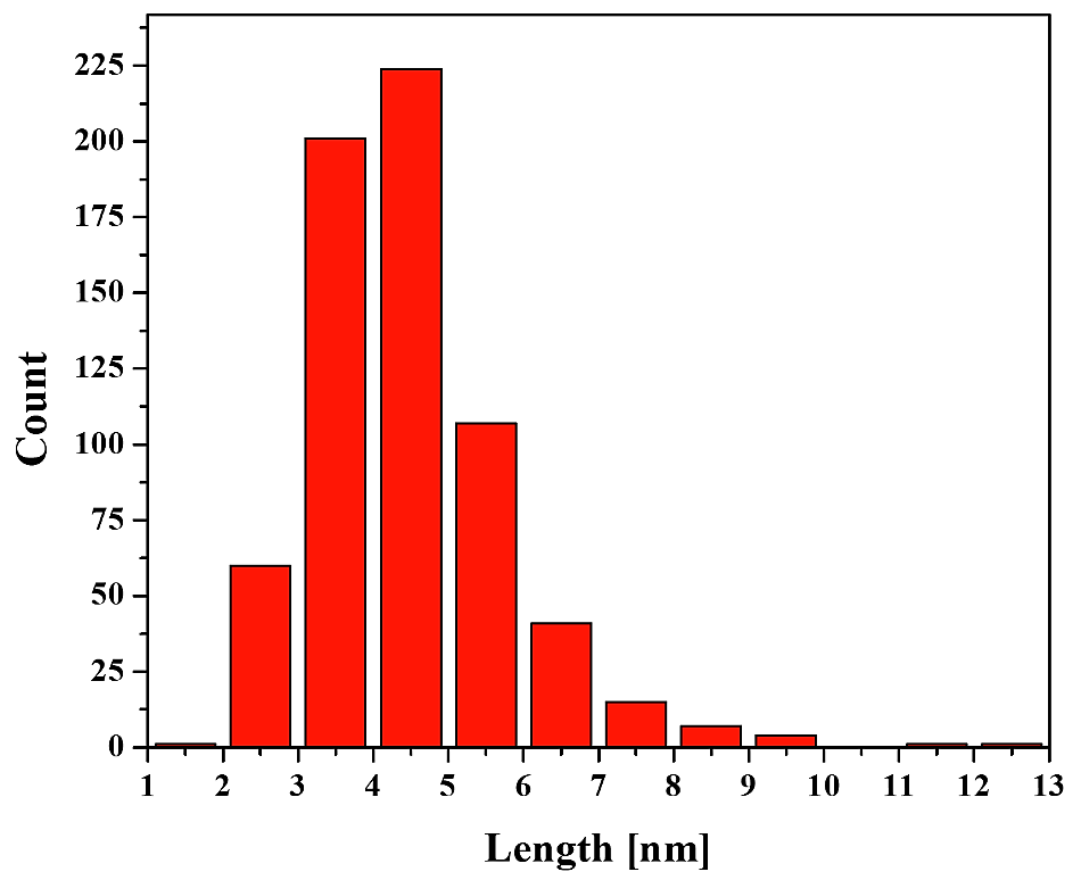

Figure S2. Frequency counts of length. 
Table S1. Optimized structures of PbTe (100) with and without $\mathrm{CH}_{2} \mathrm{O}$ as surface modifier. Height differences $\Delta \mathrm{Z}_{1}$ and $\Delta \mathrm{Z}_{2}$, to the annotated layer interacting with formaldehyde, relative to the clean optimized surface. Full optimized structures in $x y z$ format. All values annotated in $\AA$ units. Lattice parameters: $a=13.637 \AA, b=13.637 \AA, c=19.642 \AA . \alpha=\beta=$ $\gamma=90^{\circ}$.

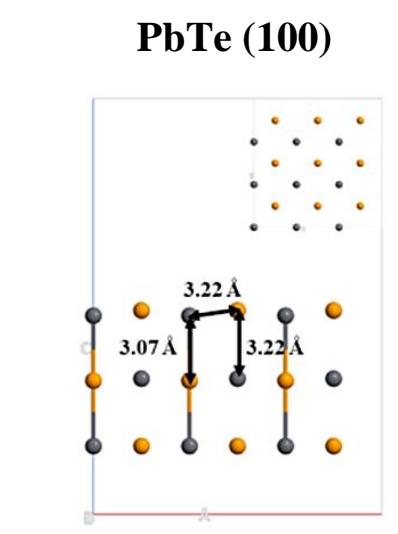

$$
\begin{aligned}
& \Delta \mathrm{Z}_{1}(\mathrm{Te}) \\
& \Delta \mathrm{Z}_{1}(\mathrm{~Pb}) \\
& \Delta \mathrm{Z}_{2}(\mathrm{Te}) \\
& \Delta \mathrm{Z}_{2}(\mathrm{~Pb})
\end{aligned}
$$

$$
\mathrm{PbTe}(100)+\mathrm{CH}_{2} \mathrm{O}
$$

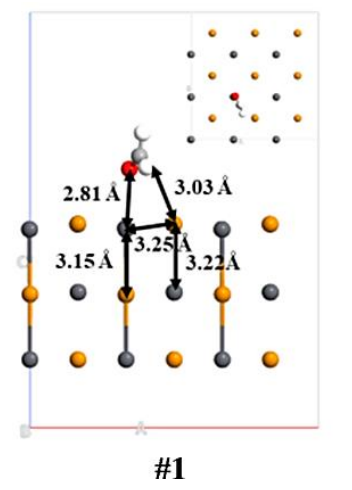

$-0.001$

0.001

$-0.001$

0.000
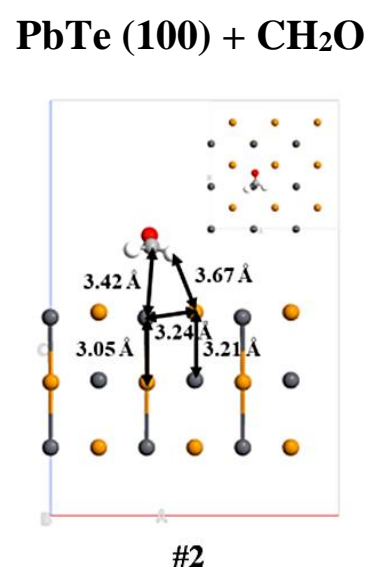

$-0.001$

0.002

$-0.001$

$-0.001$

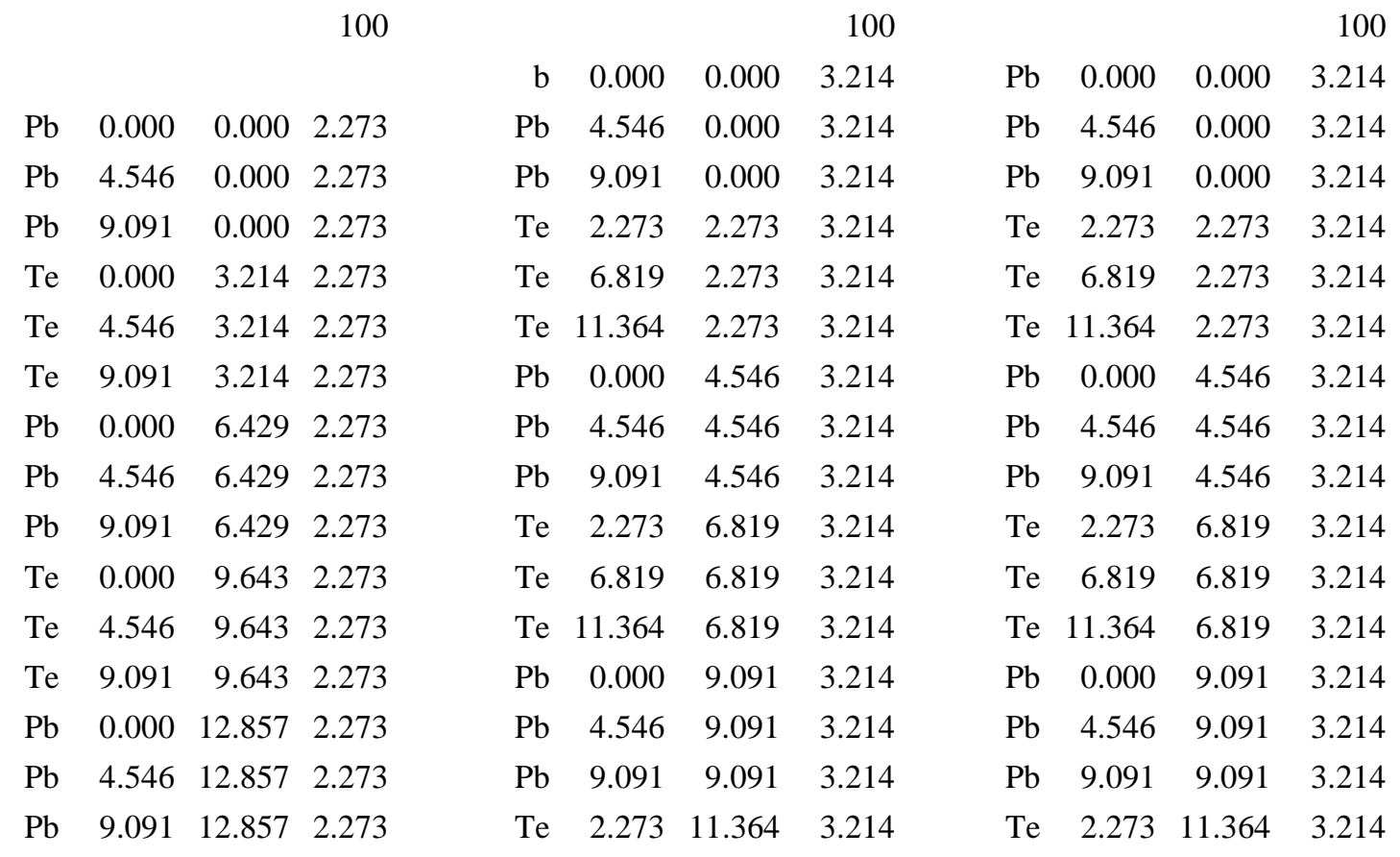




\begin{tabular}{|c|c|c|c|c|c|c|c|c|c|c|c|}
\hline $\mathrm{Te}$ & 0.000 & 16.071 & 2.273 & $\mathrm{Te}$ & 6.819 & 11.364 & 3.214 & $\mathrm{Te}$ & 6.819 & 11.364 & 3.214 \\
\hline $\mathrm{Te}$ & 4.546 & 16.071 & 2.273 & $\mathrm{Te}$ & 11.364 & 11.364 & 3.214 & $\mathrm{Te}$ & 11.364 & 11.364 & 3.214 \\
\hline $\mathrm{T}$ & 9.091 & 16.071 & 2.273 & $\mathrm{Te}$ & 0.017 & -0.001 & 6.303 & $\mathrm{Te}$ & 0.004 & -0.005 & 6.303 \\
\hline $\mathrm{T}$ & 2.273 & 0.000 & 4.431 & $\mathrm{Te}$ & 4.555 & 0.001 & 6.301 & $\mathrm{Te}$ & 4.552 & 0.006 & 6.300 \\
\hline & 6.819 & 0.000 & 4.432 & $\mathrm{Te}$ & 9.096 & -0.001 & 6.303 & $\mathrm{Te}$ & 9.089 & 0.010 & 6.302 \\
\hline $\mathrm{Te}$ & 11.364 & 0.000 & 4.431 & $\mathrm{~Pb}$ & 2.278 & 2.271 & 6.394 & $\mathrm{~Pb}$ & 2.277 & 2.275 & 6.405 \\
\hline $\mathrm{P}$ & 2.273 & 3.214 & 4.541 & $\mathrm{~Pb}$ & 6.825 & 2.271 & 6.400 & $\mathrm{~Pb}$ & 6.810 & 2.292 & 6.391 \\
\hline $\mathrm{P}$ & 6.819 & 3.214 & 4.541 & $\mathrm{~Pb}$ & 11.373 & 2.261 & 6.396 & $\mathrm{~Pb}$ & 11.373 & 2.269 & 6.400 \\
\hline $\mathrm{Pb}$ & 11.364 & 3.214 & 4.541 & $\mathrm{Te}$ & 0.005 & 4.544 & 6.301 & $\mathrm{Te}$ & 0.007 & 4.547 & 6.304 \\
\hline $\mathrm{Te}$ & 2.273 & 6.429 & 4.431 & $\mathrm{Te}$ & 4.557 & 4.552 & 6.290 & $\mathrm{Te}$ & 4.539 & 4.546 & 6.276 \\
\hline $\mathrm{T}$ & 6.819 & 6.429 & 4.431 & $\mathrm{Te}$ & 9.106 & 4.542 & 6.300 & $\mathrm{Te}$ & 9.099 & 4.547 & 6.300 \\
\hline $\mathrm{T}$ & 11.364 & 6.429 & 4.431 & $\mathrm{~Pb}$ & 2.269 & 6.821 & 6.399 & $\mathrm{~Pb}$ & 2.278 & 6.823 & 6.399 \\
\hline $\mathrm{P}$ & 2.272 & 9.643 & 4.540 & $\mathrm{~Pb}$ & 6.836 & 6.817 & 6.403 & $\mathrm{~Pb}$ & 6.809 & 6.804 & 6.402 \\
\hline $\mathrm{Pb}$ & 6.819 & 9.643 & 4.540 & $\mathrm{~Pb}$ & 11.371 & 6.817 & 6.396 & $\mathrm{~Pb}$ & 11.373 & 6.820 & 6.399 \\
\hline $\mathrm{Pb}$ & 11.365 & 9.643 & 4.540 & $\mathrm{Te}$ & 0.006 & 9.096 & 6.302 & $\mathrm{Te}$ & 0.003 & 9.098 & 6.300 \\
\hline $\mathrm{T}$ & 2.273 & 12.857 & 4.431 & $\mathrm{Te}$ & 4.557 & 9.086 & 6.311 & $\mathrm{Te}$ & 4.553 & 9.093 & 6.304 \\
\hline $\mathrm{Te}$ & 6.819 & 12.857 & 4.431 & $\mathrm{Te}$ & 9.105 & 9.097 & 6.303 & $\mathrm{Te}$ & 9.090 & 9.085 & 6.301 \\
\hline $\mathrm{Te}$ & 11.364 & 12.857 & 4.431 & $\mathrm{~Pb}$ & 2.287 & 11.355 & 6.398 & $\mathrm{~Pb}$ & 2.282 & 11.360 & 6.401 \\
\hline $\mathrm{Pb}$ & 2.273 & 16.071 & 4.541 & $\mathrm{~Pb}$ & 6.820 & 11.357 & 6.398 & $\mathrm{~Pb}$ & 6.826 & 11.363 & 6.400 \\
\hline $\mathrm{Pb}$ & 6.819 & 16.071 & 4.541 & $\mathrm{~Pb}$ & 11.369 & 11.368 & 6.395 & $\mathrm{~Pb}$ & 11.353 & 11.366 & 6.401 \\
\hline $\mathrm{Pb}$ & 11.364 & 16.071 & 4.541 & $\mathrm{~Pb}$ & -0.006 & -0.040 & 9.379 & $\mathrm{~Pb}$ & -0.013 & -0.019 & 9.379 \\
\hline $\mathrm{Pb}$ & 0.000 & 0.000 & 6.584 & $\mathrm{~Pb}$ & 4.536 & -0.019 & 9.375 & $\mathrm{~Pb}$ & 4.528 & -0.017 & 9.367 \\
\hline $\mathrm{Pb}$ & 4.545 & 0.000 & 6.584 & $\mathrm{~Pb}$ & 9.059 & -0.017 & 9.379 & $\mathrm{~Pb}$ & 9.070 & -0.014 & 9.377 \\
\hline $\mathrm{Pb}$ & 9.092 & 0.000 & 6.584 & $\mathrm{Te}$ & 2.288 & 2.276 & 9.627 & $\mathrm{Te}$ & 2.268 & 2.261 & 9.638 \\
\hline $\mathrm{T}$ & 0.000 & 3.215 & 6.820 & $\mathrm{Te}$ & 6.818 & 2.285 & 9.631 & $\mathrm{Te}$ & 6.812 & 2.269 & 9.621 \\
\hline $\mathrm{T}$ & 4.546 & 3.215 & 6.820 & $\mathrm{Te}$ & 11.378 & 2.254 & 9.608 & $\mathrm{Te}$ & 11.357 & 2.264 & 9.616 \\
\hline $\mathrm{Te}$ & 9.091 & 3.215 & 6.820 & $\mathrm{~Pb}$ & -0.025 & 4.519 & 9.376 & $\mathrm{~Pb}$ & -0.014 & 4.530 & 9.378 \\
\hline $\mathrm{P}$ & 0.000 & 6.429 & 6.584 & $\mathrm{~Pb}$ & 4.508 & 4.535 & 9.338 & $\mathrm{~Pb}$ & 4.509 & 4.558 & 9.428 \\
\hline $\mathrm{P}$ & 4.546 & 6.429 & 6.584 & $\mathrm{~Pb}$ & 9.096 & 4.515 & 9.375 & $\mathrm{~Pb}$ & 9.083 & 4.533 & 9.369 \\
\hline $\mathrm{P}$ & 9.092 & 6.429 & 6.584 & $\mathrm{Te}$ & 2.273 & 6.810 & 9.607 & $\mathrm{Te}$ & 2.250 & 6.825 & 9.615 \\
\hline $\mathrm{Te}$ & 0.000 & 9.643 & 6.820 & $\mathrm{Te}$ & 6.835 & 6.799 & 9.616 & $\mathrm{Te}$ & 6.811 & 6.809 & 9.617 \\
\hline $\mathrm{T}$ & 4.545 & 9.643 & 6.820 & $\mathrm{Te}$ & 11.376 & 6.820 & 9.610 & $\mathrm{Te}$ & 11.356 & 6.810 & 9.621 \\
\hline $\mathrm{T}$ & 9.092 & 9.643 & 6.820 & $\mathrm{~Pb}$ & -0.020 & 9.084 & 9.379 & $\mathrm{~Pb}$ & -0.026 & 9.088 & 9.375 \\
\hline $\mathrm{P}$ & 0.000 & 12.857 & 6.584 & $\mathrm{~Pb}$ & 4.536 & 9.043 & 9.395 & $\mathrm{~Pb}$ & 4.529 & 9.068 & 9.381 \\
\hline $\mathrm{P}$ & 4.546 & 12.856 & 6.584 & $\mathrm{~Pb}$ & 9.068 & 9.063 & 9.381 & $\mathrm{~Pb}$ & 9.068 & 9.067 & 9.375 \\
\hline $\mathrm{Pb}$ & 9.092 & 12.856 & 6.584 & $\mathrm{Te}$ & 2.289 & 11.356 & 9.610 & $\mathrm{Te}$ & 2.265 & 11.357 & 9.618 \\
\hline $\mathrm{T}$ & 0.000 & 16.071 & 6.820 & $\mathrm{Te}$ & 6.825 & 11.358 & 9.608 & $\mathrm{Te}$ & 6.808 & 11.357 & 9.621 \\
\hline $\mathrm{T}$ & 4.546 & 16.071 & 6.820 & $\mathrm{Te}$ & 11.366 & 11.364 & 9.610 & $\mathrm{Te}$ & 11.347 & 11.359 & 9.616 \\
\hline & 9.091 & 16.071 & 6.820 & $\mathrm{H}$ & 5.730 & 4.453 & 12.383 & $\mathrm{H}$ & 5.497 & 2.757 & 12.307 \\
\hline & & & & $\mathrm{C}$ & 4.755 & 4.877 & 12.731 & $\mathrm{C}$ & 5.173 & 3.682 & 12.833 \\
\hline & & & & $\mathrm{H}$ & 3.853 & 4.233 & 12.571 & $\mathrm{H}$ & 5.312 & 3.726 & 13.936 \\
\hline
\end{tabular}




$$
\begin{array}{llllllll}
\text { O } & 4.681 & 5.970 & 13.251 & \text { O } & 4.700 & 4.629 & 12.229
\end{array}
$$

Table S2. Optimized structures of PbTe (110) with and without $\mathrm{CH}_{2} \mathrm{O}$ as surface modifier. Height differences $\Delta Z_{1}$ and $\Delta Z_{2}$, to the annotated layer interacting with formaldehyde, relative to the clean optimized surface. Full optimized structures in $x y z$ format. All values annotated in $\AA$ units. Lattice parameters: $\mathrm{a}=13.637 \AA, \mathrm{b}=19.642 \AA, \mathrm{c}=16.818 \AA . \alpha=\beta=$ $\gamma=90^{\circ}$.

PbTe (110)

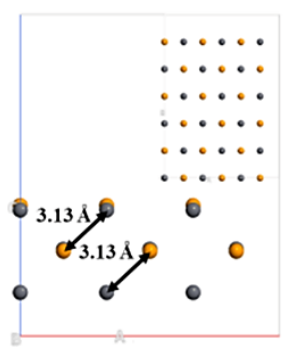

$\Delta \mathrm{Z}_{1}(\mathrm{Te})$

$\Delta \mathrm{Z}_{1}(\mathrm{~Pb})$

$\Delta \mathrm{Z}_{2}(\mathrm{Te})$

$\Delta \mathrm{Z}_{2}(\mathrm{~Pb})$
$\mathrm{PbTe}(\mathbf{1 1 0})+\mathrm{CH}_{2} \mathrm{O}$

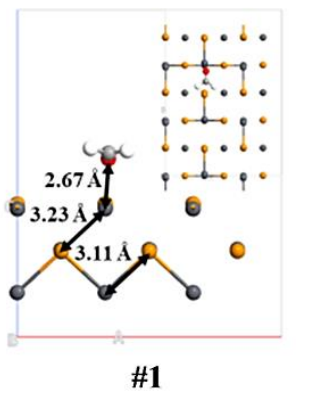

0.002

0.005

$-0.003$

$-0.004$
$\mathrm{PbTe}(110)+\mathrm{CH}_{2} \mathrm{O}$

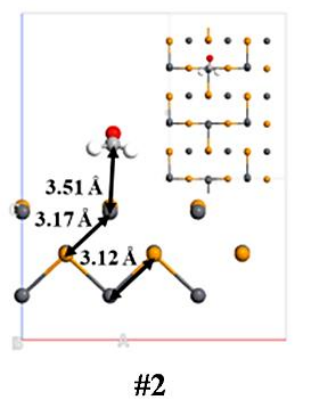

0.002

0.002

$-0.003$

$-0.003$

110

114

114

$\begin{array}{llllllllllll}\mathrm{Pb} & 0.000 & 0.000 & 2.273 & \mathrm{~Pb} & 0.000 & 0.000 & 2.273 & \mathrm{~Pb} & 0.000 & 0.000 & 2.273 \\ \mathrm{~Pb} & 4.546 & 0.000 & 2.273 & \mathrm{~Pb} & 4.546 & 0.000 & 2.273 & \mathrm{~Pb} & 4.546 & 0.000 & 2.273 \\ \mathrm{~Pb} & 9.091 & 0.000 & 2.273 & \mathrm{~Pb} & 9.091 & 0.000 & 2.273 & \mathrm{~Pb} & 9.091 & 0.000 & 2.273 \\ \mathrm{Te} & 0.000 & 3.214 & 2.273 & \mathrm{Te} & 0.000 & 3.214 & 2.273 & \mathrm{Te} & 0.000 & 3.214 & 2.273 \\ \mathrm{Te} & 4.546 & 3.214 & 2.273 & \mathrm{Te} & 4.546 & 3.214 & 2.273 & \mathrm{Te} & 4.546 & 3.214 & 2.273 \\ \mathrm{Te} & 9.091 & 3.214 & 2.273 & \mathrm{Te} & 9.091 & 3.214 & 2.273 & \mathrm{Te} & 9.091 & 3.214 & 2.273 \\ \mathrm{~Pb} & 0.000 & 6.429 & 2.273 & \mathrm{~Pb} & 0.000 & 6.429 & 2.273 & \mathrm{~Pb} & 0.000 & 6.429 & 2.273 \\ \mathrm{~Pb} & 4.546 & 6.429 & 2.273 & \mathrm{~Pb} & 4.546 & 6.429 & 2.273 & \mathrm{~Pb} & 4.546 & 6.429 & 2.273 \\ \mathrm{~Pb} & 9.091 & 6.429 & 2.273 & \mathrm{~Pb} & 9.091 & 6.429 & 2.273 & \mathrm{~Pb} & 9.091 & 6.429 & 2.273 \\ \mathrm{Te} & 0.000 & 9.643 & 2.273 & \mathrm{Te} & 0.000 & 9.643 & 2.273 & \mathrm{Te} & 0.000 & 9.643 & 2.273 \\ \mathrm{Te} & 4.546 & 9.643 & 2.273 & \mathrm{Te} & 4.546 & 9.643 & 2.273 & \mathrm{Te} & 4.546 & 9.643 & 2.273 \\ \mathrm{Te} & 9.091 & 9.643 & 2.273 & \mathrm{Te} & 9.091 & 9.643 & 2.273 & \mathrm{Te} & 9.091 & 9.643 & 2.273 \\ \mathrm{~Pb} & 0.000 & 12.857 & 2.273 & \mathrm{~Pb} & 0.000 & 12.857 & 2.273 & \mathrm{~Pb} & 0.000 & 12.857 & 2.273 \\ \mathrm{~Pb} & 4.546 & 12.857 & 2.273 & \mathrm{~Pb} & 4.546 & 12.857 & 2.273 & \mathrm{~Pb} & 4.546 & 12.857 & 2.273 \\ \mathrm{~Pb} & 9.091 & 12.857 & 2.273 & \mathrm{~Pb} & 9.091 & 12.857 & 2.273 & \mathrm{~Pb} & 9.091 & 12.857 & 2.273 \\ \mathrm{Te} & 0.000 & 16.071 & 2.273 & & & & & & & \end{array}$




\begin{tabular}{|c|c|c|c|c|c|c|c|c|c|c|c|}
\hline & 4.546 & 6.071 & 2.273 & $\mathrm{Te}$ & 0.000 & 16.071 & 2.273 & $\mathrm{Te}$ & 0.000 & 16.071 & 2.273 \\
\hline & 9.091 & 16.071 & 2.273 & $\mathrm{Te}$ & 4.546 & 16.071 & 2.273 & $\mathrm{Te}$ & 4.546 & 16.071 & 2.273 \\
\hline & 2.273 & 0.000 & 4.431 & $\mathrm{Te}$ & 9.091 & 16.071 & 2.273 & e & 9.091 & 16.071 & 2.273 \\
\hline & 6.819 & 0.000 & 4.432 & $\Gamma \mathrm{e}$ & 2.277 & -0.009 & 4.396 & e & 2.277 & 0.007 & .395 \\
\hline & 11.364 & 0.000 & 4.431 & e & 6.814 & & 4.397 & & 815 & 07 & 399 \\
\hline & 2.273 & 3.214 & 4.541 & Te & 11.364 & .040 & 4.410 & e & 11.365 & 057 & 410 \\
\hline & 6.819 & 3.214 & 4.541 & $\mathrm{~Pb}$ & 2.273 & 3.205 & 4.485 & $\mathrm{~b}$ & 2.274 & 3.223 & 4.486 \\
\hline & 11.364 & 3.214 & 4.541 & $\mathrm{~Pb}$ & 6.818 & 3.204 & 4.485 & b & 6.814 & 3.222 & 488 \\
\hline & 2.273 & 6.429 & 4.431 & $\mathrm{~Pb}$ & 11.364 & 3.241 & 4.486 & $\mathrm{~b}$ & 11.367 & 3.169 & 4.495 \\
\hline & 6.819 & 6.429 & 4.431 & $\mathrm{Te}$ & & .422 & 4.397 & $\mathrm{Te}$ & & 437 & 4.393 \\
\hline & 11.364 & 6.429 & 4.431 & $\mathrm{Te}$ & 6.814 & 6.422 & 4.396 & e & 6.815 & 6.437 & 4.396 \\
\hline & 2.272 & 9.643 & 4.540 & $\mathrm{Te}$ & 11.364 & 6.469 & 4.407 & $\mathrm{Te}$ & 11.365 & 6.372 & 4.409 \\
\hline & 6.819 & 9.643 & 4.540 & $\mathrm{~Pb}$ & 2.259 & 9.640 & 4.480 & $\mathrm{~Pb}$ & 2.276 & 9.652 & 4.492 \\
\hline & 11.365 & 9.643 & 4.540 & $\mathrm{~Pb}$ & 6.829 & & 4.484 & $\mathrm{~Pb}$ & 6.812 & & 1.495 \\
\hline & 2.273 & 12.857 & 4.431 & $\mathrm{~Pb}$ & 11.363 & 673 & 4.500 & $\mathrm{~Pb}$ & 11.367 & 9.599 & 4.494 \\
\hline & 6.819 & 12.857 & 4.431 & $\mathrm{Te}$ & 2.269 & 12.848 & 4.388 & Te & 2.273 & 12.864 & 4.389 \\
\hline & 11.364 & 12.857 & 4.431 & $\mathrm{Te}$ & 6.820 & 2.848 & 4.392 & Te & 6.818 & 12.864 & 4.394 \\
\hline & 2.273 & 16.071 & 4.541 & $\mathrm{Te}$ & 11.362 & 12.896 & 4.418 & $\mathrm{Te}$ & 11.365 & 12.801 & 4.412 \\
\hline & 6.819 & 16.071 & 4.541 & $\mathrm{~Pb}$ & & & & $\mathrm{~b}$ & & & 4.486 \\
\hline & 11.364 & 16.071 & 4.541 & $\mathrm{~Pb}$ & 6.812 & 16.056 & 4.488 & $\mathrm{~Pb}$ & 6.811 & 16.081 & 4.487 \\
\hline & 0.000 & 0.000 & 6.584 & $\mathrm{~Pb}$ & 11.365 & 16.099 & 4.476 & $\mathrm{~Pb}$ & 11.367 & 16.027 & 4.492 \\
\hline & 4.545 & 0.000 & 6.584 & $\mathrm{~Pb}$ & 0.017 & -0.254 & 6.583 & $\mathrm{~Pb}$ & 0.016 & 0.236 & 6.582 \\
\hline & 9.092 & 0.000 & 6.584 & $\mathrm{~Pb}$ & & & & $\mathrm{~Pb}$ & & & 6.599 \\
\hline & 0.000 & 3.215 & 6.820 & $\mathrm{~Pb}$ & 9.074 & -0.255 & 6.584 & $\mathrm{~Pb}$ & 079 & & 586 \\
\hline & 4.546 & 3.215 & 6.820 & $\mathrm{Te}$ & 0.004 & 3.321 & 6.825 & Te & 0.005 & 3.097 & 6.827 \\
\hline$\Gamma \mathrm{e}$ & 9.091 & 3.215 & 6.820 & $\mathrm{Te}$ & 4.546 & 3.086 & 6.841 & $\mathrm{Te}$ & 4.546 & 3.363 & 6.833 \\
\hline & 0.000 & 6.429 & 6.584 & $\mathrm{Te}$ & & 322 & 6.825 & $\mathrm{Te}$ & 086 & 00 & 6.825 \\
\hline & 4.546 & 6.429 & 6.584 & $\mathrm{~Pb}$ & 0.014 & 6.180 & & $\mathrm{~Pb}$ & & & 6.580 \\
\hline $\mathrm{Pb}$ & 9.092 & 6.429 & 6.584 & $\mathrm{~Pb}$ & 4.546 & 6.558 & 6.583 & $\mathrm{~Pb}$ & 4.543 & 6.214 & 6.592 \\
\hline $\mathrm{Te}$ & 0.000 & 9.643 & 6.820 & $\mathrm{~Pb}$ & 9.077 & 6.180 & 6.581 & $\mathrm{~Pb}$ & 9.079 & 6.664 & 6.583 \\
\hline $\mathrm{Te}$ & 4.545 & 9.643 & 6.820 & $\mathrm{Te}$ & 0.012 & 9.757 & 6.835 & $\mathrm{Te}$ & 0.006 & 9.522 & 6.830 \\
\hline & 9.092 & 9.643 & 6.820 & $\mathrm{Te}$ & & & 6.8 & $\mathrm{Te}$ & & & 6.858 \\
\hline & 0.000 & 12.857 & 6.584 & $\mathrm{Te}$ & 9.073 & 9.757 & 6.840 & $\mathrm{Te}$ & 9.085 & 9.524 & 6.828 \\
\hline $\mathrm{Pb}$ & 4.546 & 12.856 & 6.584 & $\mathrm{~Pb}$ & 0.024 & 12.623 & 6.583 & $\mathrm{~Pb}$ & 0.019 & 13.096 & 6.583 \\
\hline $\mathrm{Pb}$ & 9.092 & 12.856 & 6.584 & $\mathrm{~Pb}$ & 4.549 & 13.129 & 6.672 & $\mathrm{~Pb}$ & 4.542 & 12.670 & 6.567 \\
\hline & 0.000 & 16.071 & 6.820 & $\mathrm{~Pb}$ & 9.064 & 12.623 & 6.588 & $\mathrm{~Pb}$ & 9.075 & 13.099 & 6.588 \\
\hline & 4.546 & 16.071 & 6.820 & $\mathrm{Te}$ & 0.000 & & 6.824 & $\mathrm{Te}$ & 0.007 & 15.954 & 6.825 \\
\hline \multirow[t]{4}{*}{$\mathrm{Te}$} & 9.091 & 16.071 & 6.820 & $\mathrm{Te}$ & 4.548 & 15.997 & 6.851 & $\mathrm{Te}$ & 4.546 & 16.207 & 6.828 \\
\hline & & & & $\mathrm{Te}$ & 9.091 & 16.174 & 6.822 & $\mathrm{Te}$ & 9.084 & 15.957 & 6.822 \\
\hline & & & & $\mathrm{H}$ & 5.664 & 10.408 & 9.578 & $\mathrm{H}$ & 5.604 & 12.414 & 9.734 \\
\hline & & & & & 4.717 & 10.970 & 9.453 & $\mathrm{C}$ & 4.675 & 12.942 & 10.06 \\
\hline
\end{tabular}




$$
\begin{array}{llllllll}
\mathrm{H} & 3.780 & 10.427 & 9.691 & \mathrm{H} & 3.715 & 12.407 & 9.854 \\
\mathrm{O} & 4.710 & 12.172 & 9.162 & \mathrm{O} & 4.707 & 14.020 & 10.621
\end{array}
$$

Table S3. Optimized structures of $\mathrm{PbTe}(111)_{\mathrm{Pb}}$ with and without $\mathrm{CH}_{2} \mathrm{O}$ as surface modifier. Height differences $\Delta Z_{1}$ and $\Delta Z_{2}$, to the annotated layer interacting with formaldehyde, relative to the clean optimized surface. Full optimized structures in $x y z$ format. All values annotated in $\AA$ units. Lattice parameters: $\mathrm{a}=13.637 \AA, \mathrm{b}=13.637 \AA, \mathrm{c}=17.423 \AA . \alpha=\beta=$ $90^{\circ}, \gamma=90^{\circ}$.
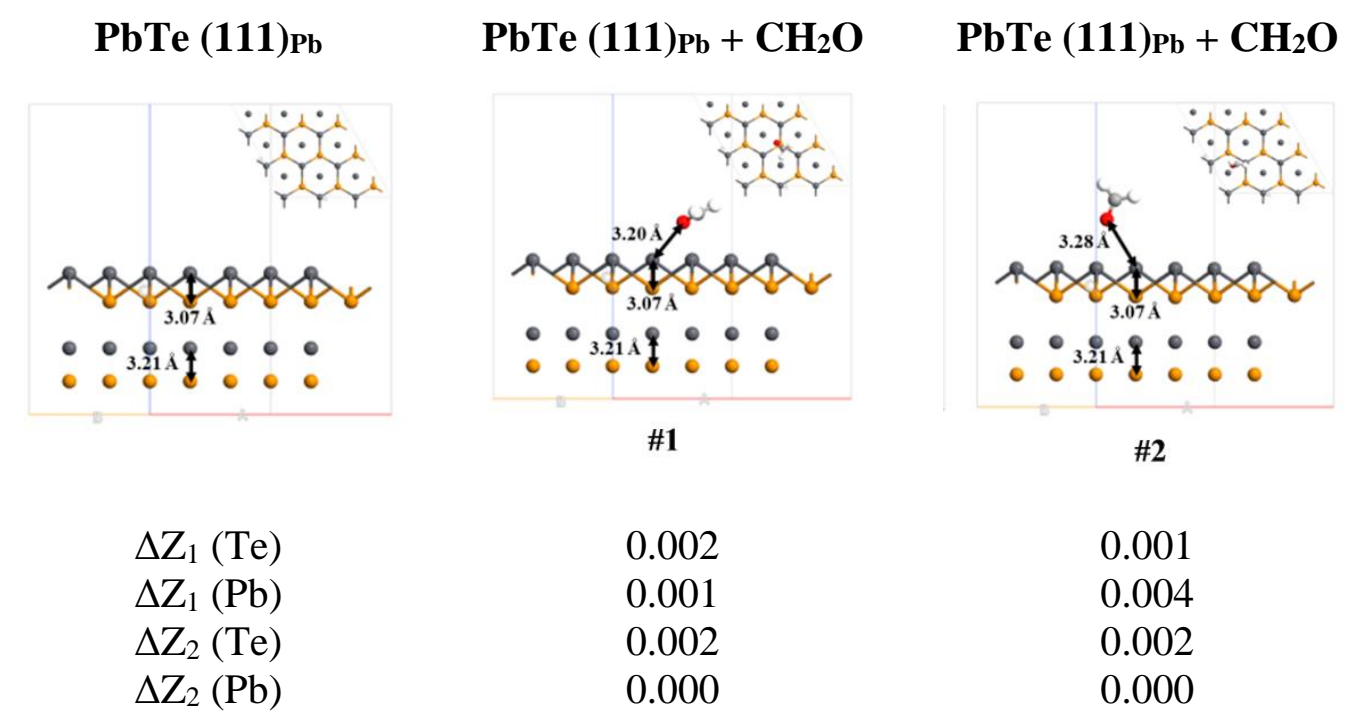
0.001
0.004
0.002
0.000

111

115.

\begin{tabular}{|c|c|c|c|c|c|c|c|c|c|c|}
\hline e 0.000 & 0.000 & 1.856 & $\mathrm{Te}$ & 0.000 & 0.000 & 1.856 & $\mathrm{Te}$ & 0.000 & 0.000 & 1.856 \\
\hline Те 4.546 & 0.000 & 1.856 & $\mathrm{Te}$ & 4.546 & 0.000 & 1.856 & $\mathrm{Te}$ & 4.546 & 0.000 & 1.856 \\
\hline e 9.091 & 0.000 & 1.856 & $\mathrm{Te}$ & 9.091 & 0.000 & 1.856 & $\mathrm{Te}$ & 9.091 & 0.000 & 1.856 \\
\hline Ге -2.273 & 3.937 & 1.856 & $\mathrm{Te}$ & -2.273 & 3.937 & 1.856 & $\mathrm{Te}$ & -2.273 & 3.937 & 1.856 \\
\hline 2.273 & 3.937 & 1.856 & $\mathrm{Te}$ & 2.273 & 3.937 & 1.856 & $\mathrm{Te}$ & 2.273 & 3.937 & 1.856 \\
\hline 6.819 & 3.937 & 1.856 & $\mathrm{Te}$ & 6.819 & 3.937 & 1.856 & $\mathrm{Te}$ & 6.819 & 3.937 & 1.856 \\
\hline-4.546 & 7.873 & 1.856 & $\mathrm{Te}$ & -4.546 & 7.873 & 1.856 & $\mathrm{Te}$ & -4.546 & 7.873 & 1.856 \\
\hline 0.000 & 7.873 & 1.856 & $\mathrm{Te}$ & 0.000 & 7.873 & 1.856 & $\mathrm{Te}$ & 0.000 & 7.873 & .856 \\
\hline 4.546 & 7.873 & 1.856 & $\mathrm{Te}$ & 4.546 & 7.873 & 1.856 & $\mathrm{Te}$ & 4.546 & 7.873 & 1.856 \\
\hline 0.000 & 2.624 & 3.712 & $\mathrm{~Pb}$ & 0.000 & 2.624 & 3.712 & $\mathrm{~Pb}$ & 0.000 & 2.624 & 3.712 \\
\hline 4.546 & 2.624 & 3.712 & $\mathrm{~Pb}$ & 4.546 & 2.624 & 3.712 & $\mathrm{~Pb}$ & 4.546 & 2.624 & 3.712 \\
\hline 9.091 & 2.624 & 3.712 & $\mathrm{~Pb}$ & 9.091 & 2.624 & 3.712 & $\mathrm{~Pb}$ & 9.091 & 2.624 & 3.712 \\
\hline-2.273 & 6.561 & 3.712 & $\mathrm{~Pb}$ & -2.273 & 6.561 & 3.712 & $\mathrm{~Pb}$ & -2.273 & 6.561 & 3.712 \\
\hline 2.273 & 6.561 & 3.712 & $\mathrm{~Pb}$ & 2.273 & 6.561 & 3.712 & $\mathrm{~Pb}$ & 2.273 & 6.561 & 3.712 \\
\hline
\end{tabular}

115 


$\begin{array}{llllllllllll}\mathrm{Pb} & 6.819 & 6.561 & 3.712 & \mathrm{~Pb} & 6.819 & 6.561 & 3.712 & \mathrm{~Pb} & 6.819 & 6.561 & 3.712 \\ \mathrm{~Pb} & -4.546 & 10.498 & 3.712 & \mathrm{~Pb} & -4.546 & 10.498 & 3.712 & \mathrm{~Pb} & -4.546 & 10.498 & 3.712 \\ \mathrm{~Pb} & 0.000 & 10.498 & 3.712 & \mathrm{~Pb} & 0.000 & 10.498 & 3.712 & \mathrm{~Pb} & 0.000 & 10.498 & 3.712 \\ \mathrm{~Pb} & 4.546 & 10.498 & 3.712 & \mathrm{~Pb} & 4.546 & 10.498 & 3.712 & \mathrm{~Pb} & 4.546 & 10.498 & 3.712 \\ \mathrm{Te} & 2.273 & 1.312 & 6.343 & \mathrm{Te} & 2.286 & 1.297 & 6.346 & \mathrm{Te} & 2.267 & 1.318 & 6.334 \\ \mathrm{Te} & 6.819 & 1.312 & 6.342 & \mathrm{Te} & 6.813 & 1.270 & 6.292 & \mathrm{Te} & 6.831 & 1.307 & 6.321 \\ \mathrm{Te} & 11.364 & 1.312 & 6.343 & \mathrm{Te} & 11.361 & 1.306 & 6.338 & \mathrm{Te} & 11.381 & 1.315 & 6.386 \\ \mathrm{Te} & 0.000 & 5.249 & 6.341 & \mathrm{Te} & 0.013 & 5.251 & 6.357 & \mathrm{Te} & -0.010 & 5.253 & 6.383 \\ \mathrm{Te} & 4.546 & 5.249 & 6.342 & \mathrm{Te} & 4.562 & 5.231 & 6.315 & \mathrm{Te} & 4.548 & 5.249 & 6.285 \\ \mathrm{Te} & 9.091 & 5.249 & 6.342 & \mathrm{Te} & 9.116 & 5.268 & 6.316 & \mathrm{Te} & 9.097 & 5.241 & 6.380 \\ \mathrm{Te} & -2.273 & 9.186 & 6.341 & \mathrm{Te} & -2.277 & 9.163 & 6.345 & \mathrm{Te} & -2.279 & 9.200 & 6.360 \\ \mathrm{Te} & 2.273 & 9.186 & 6.341 & \mathrm{Te} & 2.267 & 9.167 & 6.320 & \mathrm{Te} & 2.285 & 9.188 & 6.346 \\ \mathrm{Te} & 6.819 & 9.185 & 6.343 & \mathrm{Te} & 6.826 & 9.175 & 6.338 & \mathrm{Te} & 6.828 & 9.185 & 6.334 \\ \mathrm{~Pb} & 0.000 & 0.000 & 7.927 & \mathrm{~Pb} & -0.002 & -0.017 & 7.913 & \mathrm{~Pb} & 0.033 & -0.012 & 7.943 \\ \mathrm{~Pb} & 4.545 & -0.002 & 7.927 & \mathrm{~Pb} & 4.564 & -0.022 & 7.915 & \mathrm{~Pb} & 4.552 & 0.021 & 7.918 \\ \mathrm{~Pb} & 9.092 & -0.002 & 7.927 & \mathrm{~Pb} & 9.076 & -0.021 & 7.894 & \mathrm{~Pb} & 9.083 & -0.006 & 7.930 \\ \mathrm{~Pb} & -2.271 & 3.937 & 7.927 & \mathrm{~Pb} & -2.281 & 3.937 & 7.913 & \mathrm{~Pb} & -2.256 & 3.928 & 7.989 \\ \mathrm{~Pb} & 2.271 & 3.937 & 7.927 & \mathrm{~Pb} & 2.309 & 3.929 & 7.900 & \mathrm{~Pb} & 2.291 & 3.939 & 7.935 \\ \mathrm{~Pb} & 6.819 & 3.937 & 7.928 & \mathrm{~Pb} & 6.850 & 3.916 & 7.881 & \mathrm{~Pb} & 6.788 & 3.936 & 7.904 \\ \mathrm{~Pb} & -4.544 & 7.874 & 7.927 & \mathrm{~Pb} & -4.548 & 7.869 & 7.927 & \mathrm{~Pb} & -4.558 & 7.873 & 7.937 \\ \mathrm{~Pb} & 0.000 & 7.873 & 7.927 & \mathrm{~Pb} & 0.007 & 7.881 & 7.919 & \mathrm{~Pb} & 0.006 & 7.895 & 7.938 \\ \mathrm{~Pb} & 4.544 & 7.874 & 7.927 & \mathrm{~Pb} & 4.546 & 7.852 & 7.921 & \mathrm{~Pb} & 4.571 & 7.839 & 7.902 \\ & & & & \mathrm{H} & 3.658 & 4.797 & 11.291 & \mathrm{H} & 2.183 & 3.639 & 12.063 \\ & & & & \mathrm{C} & 4.501 & 4.595 & 10.594 & \mathrm{C} & 1.090 & 3.748 & 11.863 \\ & & & & \mathrm{H} & 4.683 & 3.525 & 10.324 & \mathrm{H} & 0.449 & 3.980 & 12.744 \\ & & & & \mathrm{O} & 5.213 & 5.493 & 10.163 & \mathrm{O} & 0.624 & 3.620 & 10.745\end{array}$

